Letters to the Editor

\title{
Retrospective Observational Study of the Environmental Impact in SARS-CoV-2 Virus Transmission based on Exclusion Criteria
}

\author{
${ }^{1 *}$ Katia Margiotti, ${ }^{1}$ Marco Fabiani, ${ }^{2}$ Marina Cupellaro, ${ }^{1}$ Alvaro Mesoraca and ${ }^{1,2,3}$ Claudio Giorlandino \\ ${ }^{1}$ ALTAMEDICA, Human Genetics Lab, Viale Liegi 45, 00198 Rome, Italy \\ ${ }^{2}$ ALTAMEDICA, Department of Biochemistry, Viale Liegi 45, 00198 Rome, Italy \\ ${ }^{3}$ ALTAMEDICA, Department of Prenatal Diagnosis, Fetal-Maternal Medical Centre, Viale Liegi 45, 00198 Rome, Italy
}

\section{Article history}

Received: 10-11-2020

Revised: 11-01-2021

Accepted: 25-01-2021

Corresponding Author:

Katia Margiotti

ALTAMEDICA, Human

Genetics Lab, Viale Liegi 45, 00198 Rome, Italy

Email: katia.margiotti@artemisia.it

\begin{abstract}
In Italy, as today October 26 the virus is circulating throughout the country. By a new decree of the Ministry of Health of October 25, 2020 , authorities in Italy have suspended most of the recreational activities, like, cinemas, theatres, gyms and restaurants. Aim of this observational study was to evaluate environmental risk related to SARS-CoV-2 infection. Information questionnaire concerning the activities carried out during the last 14 days was proposed at $256 \mathrm{SARS}-\mathrm{CoV}-2$ positive subjects. Among the SARS-CoV-2 positive subjects, 95\% (213/226) never went to cinemas and or theatres, 93\% (211/226) never went restaurants and 92\% (209/226) never went to gyms. Our data strongly suggest that the virus infection rarely occurred in the considered environmental system.
\end{abstract}

Keywords: SARS-CoV-2, Environmental Impact, COVID-19

\section{Dear Editor}

The first diagnosis of COVID-19 in Europe has been confirmed in a 38-year-old Italian healthy man. Only 4 days after the onset of COVID-19, the patient was admitted to the intensive unit care of the Policlinico San Matteo in Pavia because of respiratory failure. After several weeks of intubation and supportive treatment, the patient luckily recovered and could be discharged in good conditions. Worldwide, in less than 4 months, spread of SARS-CoV-2 virus was inevitable, has moved from affecting a few persons in Wuhan (Hubei province, China) to more than 38 million people in almost every country (Coronavirus Research Center, https://coronavirus.jhu.edu/map.html visited on October 16th, 2020) (Zhu et al., 2020; Lu et al., 2020).

In Italy for the management of Phase 2 of the emergency, a specific monitoring system on epidemiological data and response capacity of regional health services has been activated, introduced with the decree of the Ministry of Health of April 30, 2020 (http://www.salute.gov.it/portale/nuovocoronavirus/hom eNuovoCoronavirus.jsp?lingua=english). As today October 26 the virus is now circulating throughout the country. In the past recent weeks, a strong increase in cases was observed, which brought the cumulative incidence in only 14 days to 75 per 100000 inhabitants (period 28/9-11/10) versus 44,37 per 100000 inhabitants (period 21/9-4/10). In the same period, the number of symptomatic cases almost doubled 15,189 symptomatic cases in the period 28/9-11/10 versus 8,198 symptomatic cases in the period 21/9-4/10. In order to block the spreading of the virus, by a new decree of the Ministry of Health of October 25, 2020, authorities in Italy have suspended most of the recreational activity, like, cinemas, theatres, gyms and restaurants.

The aim of our retrospective observational study was to evaluate environmental risk for infection spreading related with the above-mentioned recreational activities (cinemas, theatres, gyms and restaurants) and as additional information we asked about the use of public transportation. Exclusions criteria related to environmental risk on the virus transmission could be essential in terms of decision making and economic, social and political distresses will be decreased. In the present study, we interrogated 256 SARS-CoV-2 positive subjects, that gave complete informed consent and answered to an information questionnaire concerning the activities carried out during the last 14 days. Evidence suggests that SARS-CoV-2 positivity can be detected in people 1-3 days before their symptom 
onset, with the highest viral loads observed around the day of symptom onset, followed by a gradual decline over time. The duration of SARS-CoV-2 positivity generally appears to be 14 days for asymptomatic persons and even more for patients with mild to moderate disease (He et al., 2020; Woodruff et al., 2020; Wölfel et al., 2020; Zhou et al., 2020). 5100 subject who voluntarily contacted the Altamedica diagnostic centre in Rome (COVID authorized diagnostic regional centre) from mid-September until $24^{\text {th }}$ of October 2020 were enrolled, were collected and analysed by the antigen test (STANDARD F COVID19 Ag FIA, SD Biosensor, Suwon, South Korea). Among the 5100 analysed subject, 5\% (256/5100) resulted SARS-CoV-2 virus infected, all with minimal or no symptoms, while 95\% (4844/5100) has no positive results for the antigen test. Among the 256 SARS-CoV-2 positive subjects 30 declined to answer to the information questionnaire. The sample studied are shown in Table 1. Among the SARS-CoV-2 positive subjects, $45 \%(102 / 226)$ never travel by public transportation, 95\% (213/226) never went to cinemas and or theatres, 93\% (211/226) never went restaurants and 92\% (209/226) never went to gyms (Fig. 1 and 2). Our data has shown that the infection possibly contracted during recreational activity could affect a very small percentage of SARS-CoV-2 positive subjects in our population study. Despite this is an exclusion and not an inclusion criteria study, related with the environmental risk we cannot exclude an effect on the epidemic spread related with the public transportation, since $54 \%$ of our SARS-CoV-2 positive subject reported to use public transportation assiduously and/or occasionally (Zheng et al., 2020).

Authorities in the new decree of the Ministry of Health of October 25, 2020 have limited restaurant, closed gym and others recreational activity, but they must be aware that according to the results obtained in this study $93-95 \%$ of the 226 SARS-CoV-2 positive subjects, never went to restaurant/gym or cinema. Although larger population studies are ongoing to better determine the accuracy of our consideration, data strongly suggest that the virus infection rarely occurred in the considered environmental system.

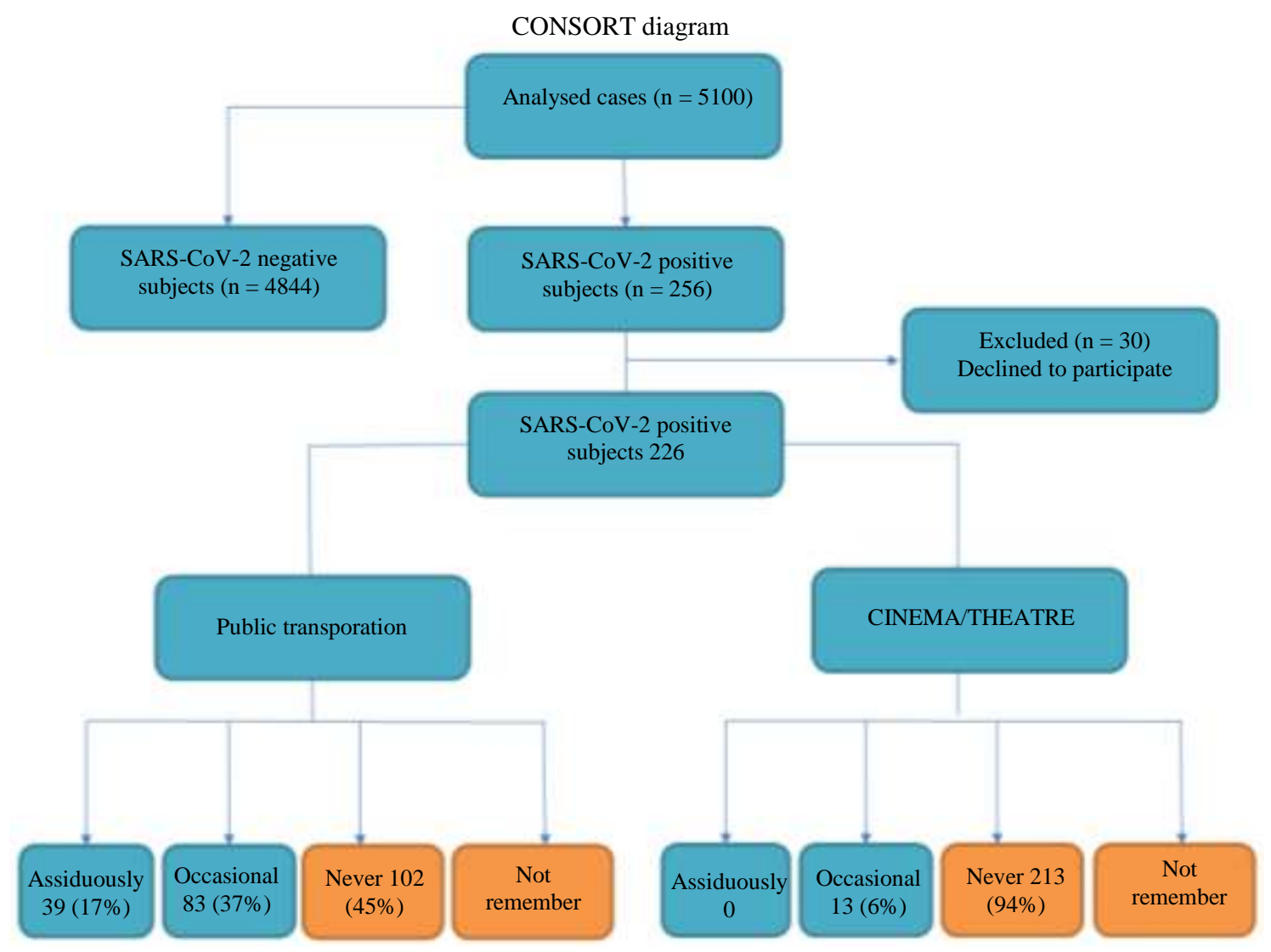

Fig. 1: From mid-September 2020, 5100 subjects were analyzed in the Altamedica laboratories, of which 4844 were negative and 256 were positive for antigen test directed towards the SAR-CoV-2 virus. Among positive subjects $93 \%$ claimed to have never attended recreational activity, such us restaurants or gyms, 14 days before contracting the virus 


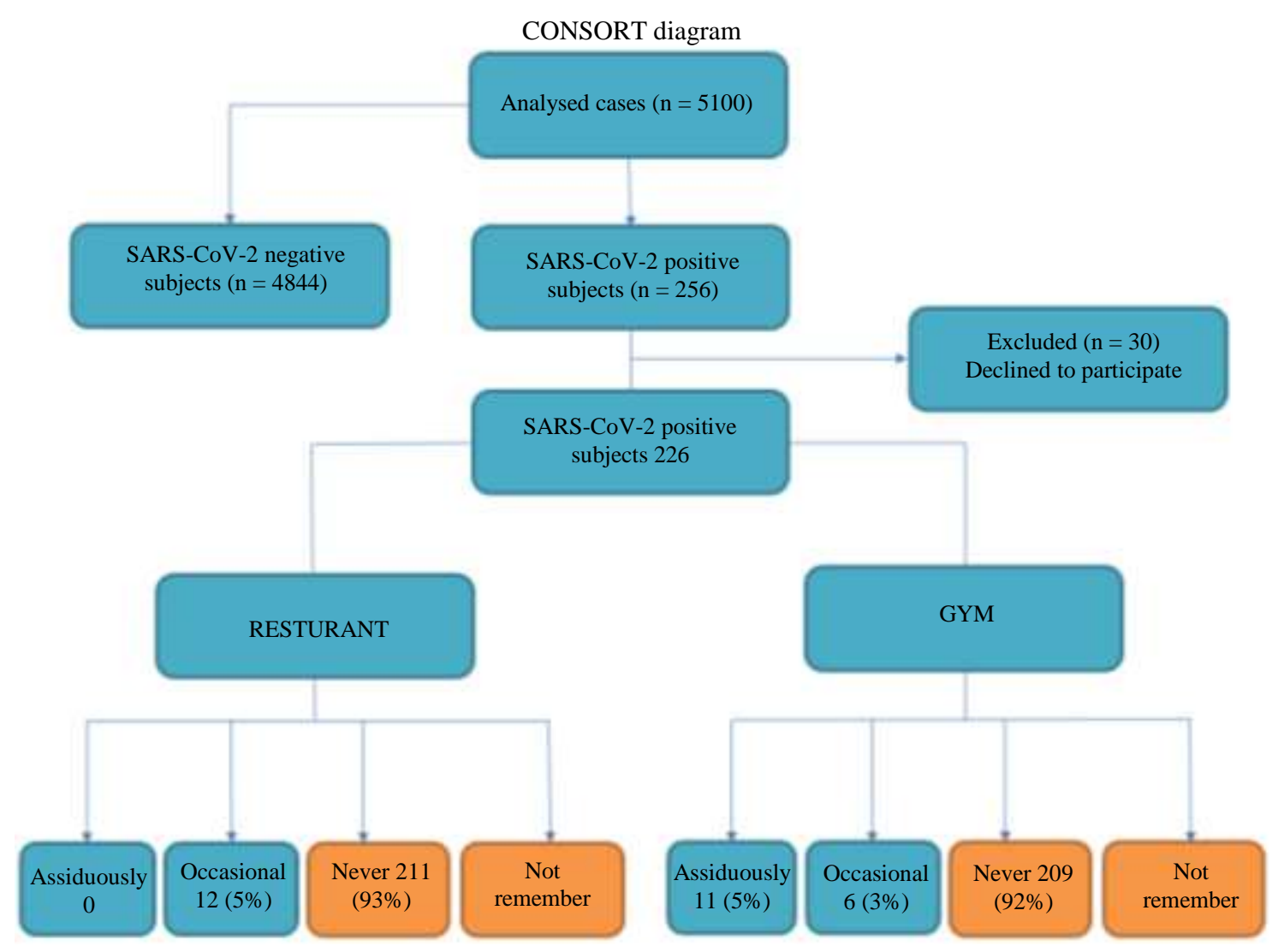

Fig. 2: From mid-September 2020, 5100 subjects were analyzed in the Altamedica laboratories, of which 4844 were negative and 256 were positive for antigen test directed towards the SAR-CoV-2 virus. Among positive subjects $95 \%$ claimed to have never attended recreational activity, such us cinemas or theatres 14 days before contracting the virus

Table 1: Patient characteristics of the COVID-19 group.

\begin{tabular}{ll}
\hline Characteristics COVID-19 group & \\
\hline Age, years & $33.5(12.5-72.4)$ \\
Male sex & $99(44 \%)$ \\
Female sex & $127(56 \%)$ \\
\hline
\end{tabular}

\section{Authors Contributions}

Katia Margiotti: Provided insights on data analysis drafting the manuscript.

Marco Fabiani: Provided insights on data analysis and revised the manuscript.

Marina Cupellaro: Performed the laboratory analysis.

Alvaro Mesoraca: Participated in conceiving the study and revised the manuscript.

Claudio Giorlandino: Conceived the study drafted and revised the manuscript.

\section{Ethics Approval and Consent to Participate}

The study was approved by the local ethical committee of Artemisia SPA and all participating patients given them written consent.

\section{Reference}

He, X., Lau, E. H., Wu, P., Deng, X., Wang, J., Hao, X., ... \& Leung, G. M. (2020). Temporal dynamics in viral shedding and transmissibility of COVID-19. Nature medicine, 26(5), 672-675.

Lu, H., Stratton, C. W., \& Tang, Y. W. (2020). Outbreak of pneumonia of unknown etiology in Wuhan, China: The mystery and the miracle. Journal of medical virology, 92(4), 401-402.

Wölfel, R., Corman, V. M., Guggemos, W., Seilmaier, M., Zange, S., Müller, M. A., ... \& Wendtner, C. (2020). Virological assessment of hospitalized patients with COVID-2019. Nature, 581(7809), 465-469.

Woodruff, A., Walsh, K. L., Knight, D., \& IrizarryAlvarado, J. M. (2020). COVID-19 infection: strategies on when to Discontinue isolation, a retrospective study. American journal of infection control, 48(9), 1032-1036.

Zheng, R., Xu, Y., Wang, W., Ning, G., \& Bi, Y. (2020). Spatial transmission of COVID-19 via public and private transportation in China. Travel Medicine and Infectious Disease. 
Katia Margiotti et al. / American Journal of Infectious Diseases 2021, 17 (1): 1.4 DOI: 10.3844/ajidsp.2021.1.4

Zhou, R., Li, F., Chen, F., Liu, H., Zheng, J., Lei, C., \& $\mathrm{Wu}, \mathrm{X}$. (2020). Viral dynamics in asymptomatic patients with COVID-19. International Journal of Infectious Diseases.
Zhu, N., Zhang, D., Wang, W., Li, X., Yang, B., Song, J., ... \& Tan, W. (2020). A novel coronavirus from patients with pneumonia in China, 2019. New England Journal of Medicine. 